\title{
Plant Growth-Promoting Rhizobacteria
}

Shrivardhan Dheeman

Potential competing interests: The author(s) declared that no potential competing interests exist.

Rhizobacteria promotes growth, immunity and manages overall health of a plants via direct and indirect mechanisms, called plant growth-promoting rhizobacteria (PGPR). 